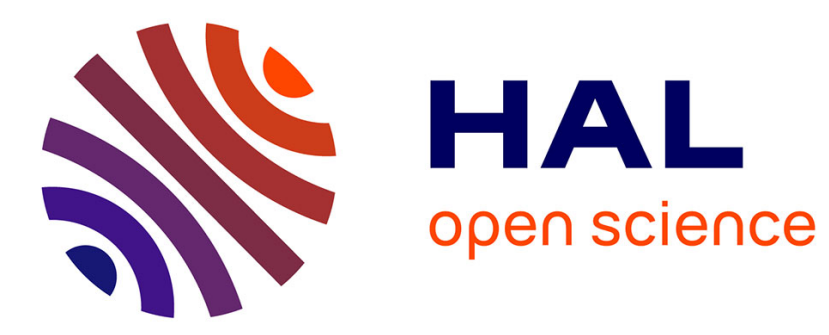

\title{
Enhancing the Stiffness Perception of Tangible Objects in Mixed Reality Using Wearable Haptics
}

Xavier de Tinguy, Claudio Pacchierotti, Maud Marchal, Anatole Lécuyer

\section{To cite this version:}

Xavier de Tinguy, Claudio Pacchierotti, Maud Marchal, Anatole Lécuyer. Enhancing the Stiffness Perception of Tangible Objects in Mixed Reality Using Wearable Haptics. IEEE VR 2018 - 25th IEEE Conference on Virtual Reality and 3D User Interfaces, Mar 2018, Reutlingen, Germany. pp.8190, 10.1109/VR.2018.8446280 . hal-01701839

\section{HAL Id: hal-01701839 \\ https://hal.inria.fr/hal-01701839}

Submitted on 6 Feb 2018

HAL is a multi-disciplinary open access archive for the deposit and dissemination of scientific research documents, whether they are published or not. The documents may come from teaching and research institutions in France or abroad, or from public or private research centers.
L'archive ouverte pluridisciplinaire HAL, est destinée au dépôt et à la diffusion de documents scientifiques de niveau recherche, publiés ou non, émanant des établissements d'enseignement et de recherche français ou étrangers, des laboratoires publics ou privés. 


\section{Enhancing the Stiffness Perception of Tangible Objects in Mixed Reality Using Wearable Haptics}

\author{
Xavier de Tinguy* \\ Univ Rennes, INSA, Inria, CNRS, \\ IRISA \\ Rennes, France
}

\author{
Claudio Pacchierotti* \\ CNRS, Univ Rennes, Inria, IRISA \\ Rennes, France
}

\author{
Maud Marchal* \\ Univ Rennes, INSA, Inria, CNRS, \\ IRISA \\ Rennes, France
}

\author{
Anatole Lécuyer* \\ Univ Rennes, Inria, CNRS, IRISA \\ Rennes, France
}

\begin{abstract}
This paper studies the combination of tangible objects and wearable haptics for improving the display of stiffness sensations in virtual environments. Tangible objects enable to feel the general shape of objects, but they are often passive or unable to simulate several varying mechanical properties. Wearable haptic devices are portable and unobtrusive interfaces able to generate varying tactile sensations, but they often fail at providing convincing stiff contacts and distributed shape sensations. We propose to combine these two approaches in virtual and augmented reality (VR/AR), becoming able of arbitrarily augmenting the perceived stiffness of real/tangible objects by providing timely tactile stimuli at the fingers. We developed a proof-of-concept enabling to simulate varying elasticity/stiffness sensations when interacting with tangible objects by using wearable tactile modules at the fingertips. We carried out a user study showing that wearable haptic stimulation can well alter the perceived stiffness of real objects, even when the tactile stimuli is not delivered at the contact point. We illustrated our approach both in VR and AR, within several use cases and different tangible settings, such as when touching surfaces, pressing buttons and pistons, or holding an object. Taken together, our results pave the way for novel haptic sensations in VR/AR by better exploiting the multiple ways of providing simple, unobtrusive, and low-cost haptic displays.
\end{abstract}

Index Terms: Human-centered computing-Human computer interaction-Interaction devices-Haptic devices

\section{INTRODUCTION}

Haptic sensation is an essential component of the user's immersive experience when interacting in Virtual or Augmented Reality. There exist many ways of simulating haptic sensations in virtual environments [4], e.g., using dedicated and actuated devices known as force feedback or tactile interfaces [13,26], using passive props also known as tangible objects [3,5,14,24], or even exploiting perceptual phenomena with cross-modal effects or sensory substitutions [2,20]. Every approach has its drawbacks and advantages, but none of them succeeds in reproducing, all-in-one, the complex richness of real haptic exploration, especially in a simple and cost-effective manner.

In this paper, we are interested in improving haptic displays in virtual environments by taking the best of two simple haptic solutions: tangible objects and wearable haptics. On the one hand, tangible objects are known to be very effective at providing global and distributed shape sensations. However, being often passive, tangible objects are usually unable to simulate several varying contact sensations. On the other hand, wearable haptics is gaining more and more interest in VR/AR, being unobtrusive, lightweight, inexpensive, and able to display varying touch sensations when interacting with virtual objects. However, these wearable devices are usually not able to provide kinesthetic feedback, failing at effectively simulating stiff contacts and global shapes.

*e-mail: \{xavier.de-tinguy, claudio.pacchierotti, maud.marchal, anatole.lecuyer\}@irisa.fr

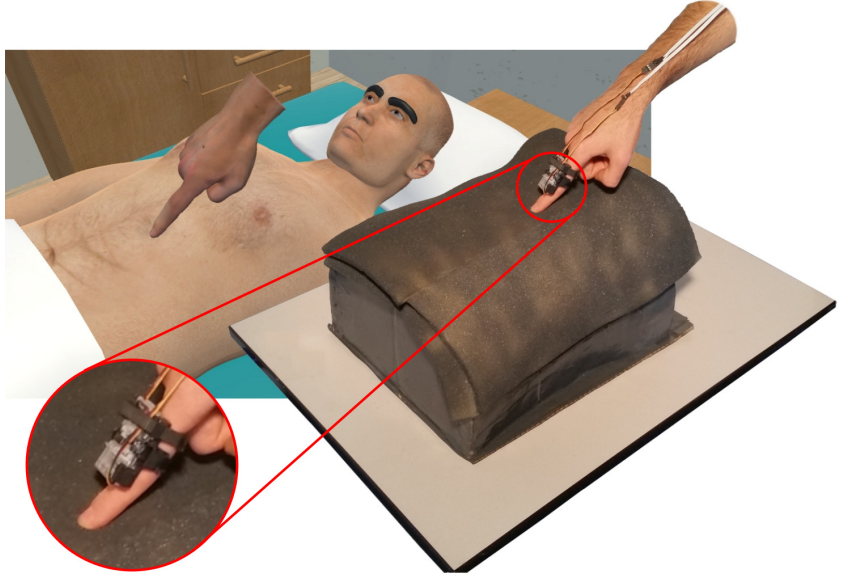

Figure 1: Our approach implemented in a VR medical palpation simulator. We propose an innovative approach for improving haptic sensations in VR/AR applications, capable of dynamically changing the perceived stiffness of tangible objects by providing timely tactile stimuli through wearable haptic devices. Passive tangible objects (a tangible chest here) provide haptic information about the global shape/percept of the virtual objects, while wearable haptic devices provide haptic information about dynamically changing mechanical properties (local elasticity here).

Therefore, to improve the range and effectiveness of haptic sensations in virtual environments, we study the effect of combining tangible objects (for simulating the global and distributed shape/percept of the virtual object) together with wearable haptics (for dynamically changing the mechanical properties of the object). This idea is illustrated in Fig. 1. In this work, we focus on altering the sensation of stiffness/elasticity of virtual/tangible objects, since it is a prominent tactile feature and it is relevant for many applications. For example, in robotics, increasing the stiffness of virtual and remote environments through a combination of kinesthetic and tactile stimuli has shown to increase the stability and safety of teleoperation systems [27].

The main contributions of our work can be summarized as follows:

- we proposed a novel approach for enhancing the stiffness perception of tangible objects through wearable haptics;

- we designed a proof-of-concept system meant to render various sensations of stiffness for tangible objects in AR or VR, relying on a 2-DoF wearable tactile display for the finger;

- we conducted a user study assessing the possibility to alter the perceived stiffness of real objects using wearable haptic stimulation, as well as studying the importance of the locus of this stimulation (in other words, we wanted to answer the question: "can we provide the tactile sensation away from the fingertip - enabling user's direct interaction with objects retaining our stiffness alteration effect?"); 
- we designed several settings and use cases in virtual and/or augmented reality, in order to illustrate the potential of our approach in different contexts, e.g., medical palpation, industrial training, entertainment.

\section{RELATED WORK AND CURRENT LIMITATIONS}

Tangible objects. Tangible objects are known to be very effective at providing human users with haptic information about distributed shape and weight sensations. Interacting with tangible objects has been proven to significantly improve the immersiveness of VR and AR systems, even when providing no additional haptic feedback [3, 5, 14, 24]. For example, Billinghurst et al. [3] presented an AR tangible book. Users can turn its pages, look at the pictures, and read the text as in a normal book. However, if they use an AR display, they can also see 3-dimensional virtual models popping out of the pages. Users can then change the models by physically turning the book pages. AR2 Hockey [24] is another example of AR game using tangible objects. It enables two users to hit a virtual puck on a real table. In all the above mentioned cases, the tangible objects were made out of relatively inexpensive materials and were not able to actively change their mechanical properties, e.g., their shape or weight. To address this issue, researchers started developing actuated tangible objects able to actively change as the user interacts with them. For example, systems such as inFORM [8] and TRANSFORM [15] enable experimentation with shape-changing interfaces and dynamic physical affordances; alternatively, Zhao et al. [31] used self-assembling robots to form tangible objects shaped like the considered virtual object. More recently, Harley et al. [12] presented a system for diegetic tangible objects in VR. They developed four tangible objects prototypes, including a furry, hollow raccoon toy, which had a skeletal cage to give structural integrity to its body and a heartbeat that could be either calm or accelerated.

Although quite effective, the haptic feedback provided by the aforementioned systems is actively delivered through the tangible object, and developing multiple, ad-hoc, haptic-enabled tangible objects for each considered scenario requires a significant amount of work. Moreover, active tangible objects can be very expensive to build.

Finger worn haptic devices. Popular techniques to provide rich wearable haptic feedback in the literature are through moving platforms, that can orient and/or translate on the skin, pin-arrays, shearing belts and tactors, pneumatic jets, and balloon-based systems. For example, Minamizawa et al. [23] presented a wearable fingertip device consisting of two DC motors that move a belt in contact with the user's fingertip. When the motors spin in opposite directions, the belt presses into the user's fingertip, and when the motors spin in the same direction, the belt applies a tangential force to the skin. More recently, Girard et al. [10] developed a wearable fingertip device capable of rendering 2-DoF skin stretch stimuli.Two DC motors move a tactor in contact with the finger pulp, achieving a maximum displacement of $2 \mathrm{~mm}$ in both directions. A review on wearable haptic devices for the hand can be found in [26].

Wearable haptics has a great potential in the fields of virtual and augmented reality. In fact, many of the devices described in [26] have been evaluated and tested in VR. For example, the wearable haptic device by Girard et al. [10] was tested in several user cases in VR, like tapping on a virtual bottle, feeling the texture of a virtual surface, and feeling the weight of a virtual object. A device similar to Minamizawa et al. [23] was also used by Pacchierotti et al. [25] in a pick-and-place VR experiment. While using wearable haptic devices in VR has become quite popular, it is rarer to see them used in AR. As mentioned before, this is mostly due to the fact that many wearable haptic devices are designed to be worn on the fingertips [26], preventing users to interact with the real environment. One of the most recent examples of wearable haptics for AR is the work of Maisto et al. [22]. They presented the experimental evaluation of two wearable haptic interfaces for the fingers in AR.
The first one is a 3-DoF fingertip device, which applies tactile stimuli through a rigid moving platform; the second one is a $2-\mathrm{DoF}$ skin stretch device for the finger, which is similar to [23] and applies tactile stimuli through a soft belt.

Mixing tangible objects and haptic devices. Developing multiple, ad-hoc, haptic-enabled tangible objects for each considered scenario requires a significant amount of work and may also be rather expensive. In this respect, researchers have worked towards finding alternative, simpler solutions to the problem of providing varying contact sensations. For example, pseudo-haptics uses vision to distort haptic perception, and it has been used to alter various haptic properties such as the stiffness of a virtual spring, the texture of an image, or the mass of a virtual object $[16,20]$.

Another promising approach to vary the mechanical properties of tangible objects is interacting with them through external hapticenabled tools. For example, Harders et al. [11] presented a multimodal AR setup in which a leg dummy is augmented with virtual soft tissue. Haptic feedback is generated through a grounded PHANToM 1.5 and the visual augmentation is provided through a custom head-mounted display. Users interacted with the dummy through the end-effector of the PHANToM interface, feeling the combination of forces due to the contact with the dummy and the haptic feedback provided by the grounded interface. Using a similar approach, Jeon et al. [17, 18] augmented a breast silicon module with a virtual tumor. More recently, Pacchierotti et al. [27, 28] used a wearable fingertip tactile device to increase the perceived stiffness of a virtual environment rendered by a grounded kinesthetic interface. Increasing the stiffness of a virtual or remote environment through tactile haptics is a promising approach in robotic scenarios as it guarantees the passivity of the system [27]. Also Park et al. [29] used tactile stimuli to increase the perceived stiffness of a virtual environment rendered by a grounded kinesthetic interface. Although effective, these approaches make use of grounded interfaces, which are often very expensive and have a rather limited workspace. Culbertson and Kuchenbecker [7] presented an ungrounded haptic augmented reality system that alters the roughness and friction of a rigid threedimensional object. The user touches the object via a custom haptic stylus. A textural roughness model generates a vibration waveform in real time, which is played through a voice coil actuator. A solenoid inside the stylus applies a braking force to the ball for friction rendering. A similar approach using a wearable vibrotactile ring has been presented in $[1,21]$. Minamizawa et al. [23] increased the perceived weight of tangible objects using skin stretch stimuli provided by a wearable device.

In this paper, we aim at combining tangible objects and wearable haptics, so that the haptic interaction is not mediated by any external tool and the user's fingertip can directly contact the tangible surface. Since the relationship between applied force and finger displacement contributes to the perception of stiffness [30], we increased the perceived stiffness of tangible objects by providing additional timely pressure stimuli on the finger's skin. To provide such stimuli, we used a 2-DoF wearable tactile device for the finger, similar to [23, 25].

We believe that the use of wearable haptics can enable a promising combination of both passive haptic feedback, delivered by uncomplicated and inexpensive tangible objects, and active haptic feedback, delivered by one or more wearable haptic devices. Tangible objects can indeed help deliver those haptic sensations that wearable haptic devices cannot provide. In fact, wearable haptic devices can usually only provide ungrounded tactile stimuli (e.g., local shape, texture) and most kinesthetic sensations (e.g., weight, general shape) are missed [13]. Moreover, wearable devices can only provide stimuli to a reduced number of contact points (e.g., the fingertips), and increasing the number of these points directly affects the wearability and comfort of the system [26]. On the other hand, passive tangible objects cannot provide varying contact sensations, severely affecting their applicability in VR and AR scenarios. 


\section{PRoOf of CONCEPT: AUgMENTING tANGIBLE OBJECTS STIFFNESS USING A FINGER WEARABLE TACTILE DEVICE}

In order to validate the effectiveness and viability of the proposed approach, we started by addressing a proof-of-concept scenario aiming at increasing stiffness sensations at the fingers. Toward this, we built a suitable wearable haptic device. Wearability, comfort, effectiveness, ease of use, and inexpensiveness are the foremost requirements we considered in the choice of the proposed device. Below we describe the wearable haptic device and a first experience which describes our idea.

\subsection{2-DoF wearable haptic device}

We built a custom 2-DoF wearable haptic device capable of providing pressure and skin stretch stimuli at the skin, shown in Fig. 2. Its actuation principle has been inspired by the device presented by Minamizawa et al. [23], while its design has been inspired by the "hRing" device presented by Prattichizzo's group at the University of Siena [25]. It is composed of a static structure, housing two servo motors, and a fabric belt, that applies the requested stimuli to the skin. A Velcro strap band is used to secure the device on the finger When the two servo motors rotate in opposite directions, the belt is pulled up or down, providing a varying force normal to the finger. On the other hand, when motors spin in the same direction, the belt applies a shear force to the finger. To adjust the device for different finger sizes, we built eight finger-device adapters, enabling us to adapt the size of our device in less than 15 seconds. The device weights $17 \mathrm{~g}$ for $42 \times 22 \times 33 \mathrm{~mm}$, and it can be worn at the fingertip as well as at the middle or proximal phalanges.

Since the servomotors are position controlled, it is only possible to command them with a desired angle. The relationship between the commanded angle and belt displacement for each motor is $\Delta b_{i}=$ $r \Delta \theta_{i}, i=1,2$, where $r=5 \mathrm{~mm}$ is the radius of the servo motor pulley, $\Delta b_{i}$ the commanded belt displacement due to the motion of motor $i$, and $\Delta \theta_{i}$ the $i$-th motor commanded angle expressed in radians. In our case, the two motors always rotate of the same amount, i.e., $\left|\Delta \theta_{1}\right|=\left|\Delta \theta_{2}\right|$ and $\left|\Delta b_{1}\right|=\left|\Delta b_{2}\right|$. Moreover, we only consider stiffness sensations and, therefore, we will always move the motors in opposite directions (as in Fig. 2b). Nonetheless, this 2-DoF design will enable us to quickly move toward testing, in the near future, the effectiveness of our approch for other types of tactile sensations. Finally, we can also relate the total vertical belt displacement $\Delta b_{s}=\operatorname{sgn}\left(\Delta \theta_{2}\right) \Delta b_{2}$ to the normal force applied by the belt on the finger skin, $f_{\text {tact }}=k_{\text {skin }} \Delta b_{s}$, where $k_{\text {skin }}$ is the finger stiffness value. In this work, the maximum displacement range of the device was $6 \mathrm{~mm}$ and we considered an isotropic elastic behavior with $k_{\text {skin }}=0.5 \mathrm{~N} / \mathrm{mm}[6,25]$.

Since this device cannot provide the sensation of making/breaking contact with the virtual environment (i.e., the belt always contact the skin), the contact area between the end-effector and the finger skip is constant. For this reason, in this work we do not consider the effect of the temporal change of contact area in the perception of stiffness (see also Sect. 6).

\subsection{Proof-of-concept demonstrator: increasing the stiff- ness of a foam as it is pressed}

We now introduce our approach through a preliminary but representative prototype demonstrator. Sect. 4 will then carry out a humansubject study, to understand and quantitatively measure how to alter the perceived stiffness of tangible objects using wearable haptic stimulation, as well as addressing the importance of the locus of stimulation (e.g., fingertip vs. middle phalanx vs. proximal phalanx).

The setup is shown in Fig. 3. The human user wears one wearable tactile device on the right index finger. We chose to place the wearable device on the proximal finger phalanx, in order to leave the fingertip free to interact with the tangible environment. The implications of wearing the device on the proximal phalanx instead of the fingertip are the focus of Sect. 4. In front of the user, on top of a table,

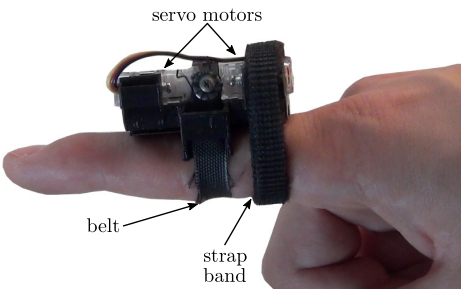

(a) Prototype (side view).

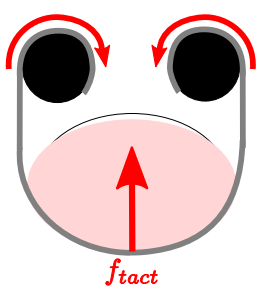

(b) Actuation principle (front view).
Figure 2: 2-DoF wearable haptic device used in our proof-ofconcept [25]. When the motors rotate in opposite directions, the belt provides a varying pressure stimuli to the finger; when motors spin in the same direction, the belt applies a shear force to the finger.

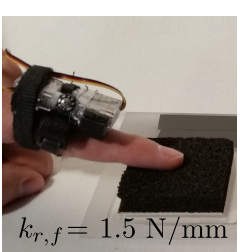

(a) The setup.

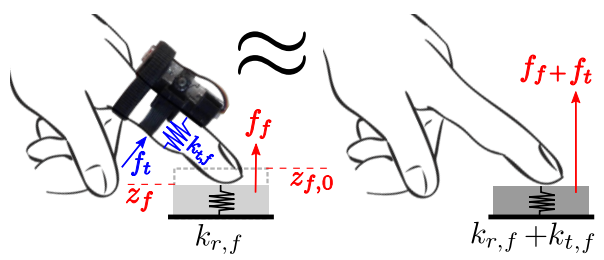

(b) The wearable tactile device increases the perceived stiffness of the foam every time it is pressed.
Figure 3: Proof-of-concept demonstrator. Users wear the wearable device on their index finger and interact with the foams. By providing timely tactile sensations through our wearable device, we want to increase the perceived stiffness of the foam as the user presses it.

we placed a small board with a foam of stiffness $k_{r, f}=1.5 \mathrm{~N} / \mathrm{mm}$ (see Fig. 3a). This value was empirically derived modeling the foam as a spring system. Users can then repeatedly press the foam with the finger wearing the device. When compressed, the foam provides the user with a force $f_{f}=-k_{r, f}\left(z_{f}-z_{f, 0}\right)$, where $z_{f}$ is the height of the foam when compressed and $z_{f, 0}$ is its height when no load is applied (see Fig. 3b).

Our hypothesis is that, by providing timely tactile sensations through our wearable device, we can increase the perceived stiffness of the foam as the user presses it, making it feel stiffer and stiffer every time it is pressed. Specifically, we speculate that we can increase the perceived stiffness of the foam of $0.1 \mathrm{~N} / \mathrm{mm}$ every time it is pressed. This concept is sketched in Fig. 3b: every time the user touches the foam, the wearable device provided a timely additional tactile force, aimed at increasing the foam perceived stiffness. This additional tactile force $f_{t}$ considers again a spring model, $f_{t}=-k_{t, f}\left(z_{f}-z_{f, 0}\right)$, where $k_{t, f}$ is the additional stiffness we want the user to perceive. In our case, $k_{t, f}=0 \mathrm{~N} / \mathrm{mm}$ the first time the user touches the foam and it increases of $0.1 \mathrm{~N} / \mathrm{mm}$ every time the foam is pressed.

\section{Perceptual evaluation}

We performed a perceptual evaluation that aims at assessing the capability of our wearable device to increase the perceived stiffness of real objects. We also wanted to evaluate the effect of moving the haptic stimuli away from the point of contact with the real environment. Our hypotheses are:

H1. providing timely tactile stimuli through our wearable device makes the users perceive a real object stiffer than it actually is

$\mathrm{H} 2 . \mathrm{H} 1$ is still valid even when providing these tactile stimuli far away from where the contact with the real object happens (i.e., the fingertip). 


\subsection{Experimental apparatus and participants}

To study how the perception of stiffness of a real object is influenced by the wearable device, we conducted an experiment in which participants could interact with a piston-like tangible object representing our 1D stiffness. The setup is shown in Fig. 4. Subjects were asked to wear the wearable tactile device on their finger at different positions and look at a 52-cm-diagonal LCD screen showing the virtual scene. A 3-DoF grounded Falcon haptic interface (Novint Technologies, USA) was placed next to them, with its end-effector facing upwards and a tangible piston-like object fixed at the top. To avoid sliding, the tangible object was covered by a thin layer of rubber. Participant could feel different stiffness rendered by the Falcon when pressing on the tangible object with their right index fingertip At the same time, additional tactile stimuli could be generated by the wearable device or not. Since the tangible piston-like was not too compliant, the induced temporal change of the contact area, when pressing pistons of variable stiffness, was almost constant. To avoid any crossmodal effect, participants were isolated from external noise through a pair of headphones playing white noise.

Sixteen participants ( 13 males, 3 females, $M=25.44, S D=5.08$ ) took part to the experiment, all of whom were right-handed. Three of them had previous experience with haptic interfaces.

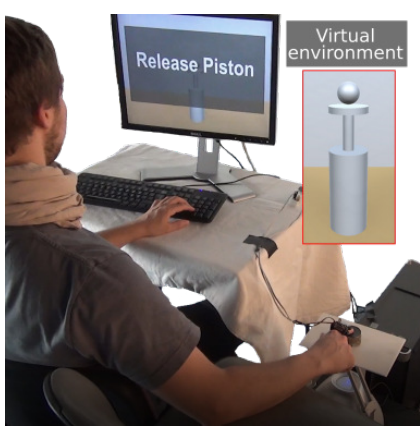

(a) Overview of the experimental setup.

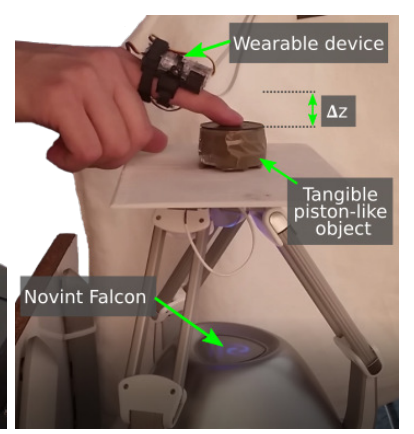

(b) Detail of the interaction with the tangible object.
Figure 4: Perceptual evaluation. The Falcon haptic interface simulates the interaction with a tangible $1-\mathrm{DoF}$ piston with variable stiffness. Whenever the user presses on the Falcon interface, the virtual piston moves accordingly.

\subsection{Procedure}

As shown in Fig. 4a, the virtual scene is composed of a piston, whose position is linked to the position of the Falcon's end-effector.

Participants had to compare two pistons with different rendered stiffness, modeled by a 1D spring law: $f=-k \Delta z$ if $\Delta z>0 \mathrm{~mm}$, $0 \mathrm{~N}$ otherwise, where $\Delta z$ is the difference between the position of the Falcon's end-effector and the resting position of the piston (see Fig. 4b). Subjects were asked to interact with a first piston for $2 \mathrm{~s}$ after the first press, and then to move their fingertip away from the end-effector to enable its release. After that, they were asked to interact in a similar way with a second piston. After this second interaction, participants were finally asked to judge which of the two pistons felt stiffer. One piston served as a reference, displaying a reference stiffness $k_{\text {ref }}$ provided only by the Falcon, while the other piston displayed a variable stiffness $k_{\text {test }, F}+k_{\text {test }, T}$ provided using both the Falcon and the wearable tactile device, respectively. The test stiffness $k_{t e s t, T}$ provided by the wearable device was constant in all the conditions, while the test stiffness $k_{t e s t, F}$ provided by the Falcon changed, as detailed below.

After preliminary testings, we considered 6 values of stiffness $k_{\text {test }, F}$ to be compared with the stiffness of the reference piston $k_{r e f}=0.1 \mathrm{~N} / \mathrm{mm}$. The six values of the test piston were: $-30 \%$,
$-15 \%,-7.5 \%,+7.5 \%,+15 \%$ and $+30 \%$ of the reference stiffness. In these six conditions, the wearable device always rendered the same additional stiffness $k_{\text {test }, T}$.

The experimenter explained the procedures and spent about 2 minutes adjusting the setup to be comfortable before the subject began the experiment. The experiment lasted 50 minutes in total.

\subsection{Conditions and plan}

Three conditions are considered in our experimental design:

- $\mathbf{C 1}$ is the difference of stiffness between the reference piston and the test piston, $\left|k_{r e f}-k_{t e s t}\right|$. As mentioned before, three differences were possible: $0.0300 \mathrm{~N} / \mathrm{mm}, 0.0150 \mathrm{~N} / \mathrm{mm}$ and $0.0075 \mathrm{~N} / \mathrm{mm}$, corresponding to the absolute values of the difference of the six possible stiffness of the test piston with the stiffness of the reference piston.

- C2 corresponds to a binary variable, which is true if the piston perceived as the stiffest is the one manipulated when the wearable device is active.

- C3 is the position of our wearable device on the participant finger. Four possible positions were chosen: three on the finger pushing on the cardboard (Proximal, Middle and Fingertip of the right hand), and one on the index fingertip of the left hand of the participant.

The order of presentation of the two pistons and the order of the finger positions were counterbalanced to avoid any order effect: every couple of pistons was therefore presented in all orders. Thus, participants were presented with 140 trials, divided in 4 blocks (C3) of 35 trials in a different randomized order for each block. Each block of 35 trials presented a set of couples of pistons made of 7 stiffness values (C1) with 5 trials for each.

\subsection{Collected Data}

For each couple of piston, we collected as an objective measure the participant's answer. This answer corresponds to the piston (first or second) which was reported by the participant as the stiffest. The measure was then collected as a true discovery rate, i.e., if the answer corresponds to the stiffest value rendered.

Participants also completed a subjective questionnaire at the end of the experiment. Each question of this questionnaire was answered using a 7-item Likert scale:

- Q1: It felt like pressing a real piston.

- Q2: The haptic device on your finger contributed to the perception of stiffness.

- Q3: The combination of both cutaneous and kinesthetic sensations contributed to the perception of stiffness.

- Q4: The tactile device provides a higher contribution to the perception of stiffness.

- Q5: Practicing improves the association of both cutaneous and kinesthetic sensations.

- Q6: The locations of the tactile feedback did not influence my perception of stiffness.

- Q7: After the experiment, I felt tired.

\subsection{Results}

Recognition rate of the stiffest piston. To study the recognition rate of the stiffness in function of the three conditions, we used a logistic regression model on the collected data to model the probability of recognition of the stiffest piston with respect to the three independent variables $\mathbf{C 1}, \mathbf{C 2}$ and $\mathbf{C 3}$ defined in the experimental design. The participants are considered as a random effect in the model.

We performed an analysis of deviance of the logistic regression model and we found a significant marginal effect for both C1 $(p<0.001)$ and C2 $(p<0.001)$, as well as an interaction effect between $\mathbf{C 2}$ and $\mathbf{C 3}(p=0.016)$. 
We performed a post-hoc analysis on the condition $\mathbf{C 1}$ using a Tukey test adapted to the logistic generalized regression model. We found that all the differences of stiffness between the reference and test pistons were significant $(Z=4.19, Z=9.14, Z=5.27$ for the differences between 0.015 and $0.0075,0.03$ and 0.0075 , and 0.03 and 0.015 respectively, and $p<0.001$ for the three differences).

Concerning the interaction effect between the $\mathbf{C 2}$ and $\mathbf{C 3}$, we performed a pairwise comparison on all the pairs based on least squares means estimates. Figure 5 shows, for each of the four different positions on the fingers, the probability of finding the stiffest piston in function of the differences of stiffness (C1). The plot distinguished whether the piston with the activated wearable device was considered as the stiffest or not (C2). Both the data and the model built from the analysis are shown. The probability of finding the stiffest piston differs when the wearable device is activated or not, in function of the wearable device position on the finger. There is a significant difference between the left finger tip position and the others $(Z=2.82$ and $p=0.028$ for the right finger tip, $Z=2.31$ and $p=0.042$ for the middle position, and $Z=2.55$ and $p=0.033$ for the proximal position). As shown in Figure 5, the difference between the probability of finding the stiffest piston when the stiffest piston is the one with the wearable device and when the stiffest piston is the reference is smaller for the left finger tip position (blue curve) than for the other positions.

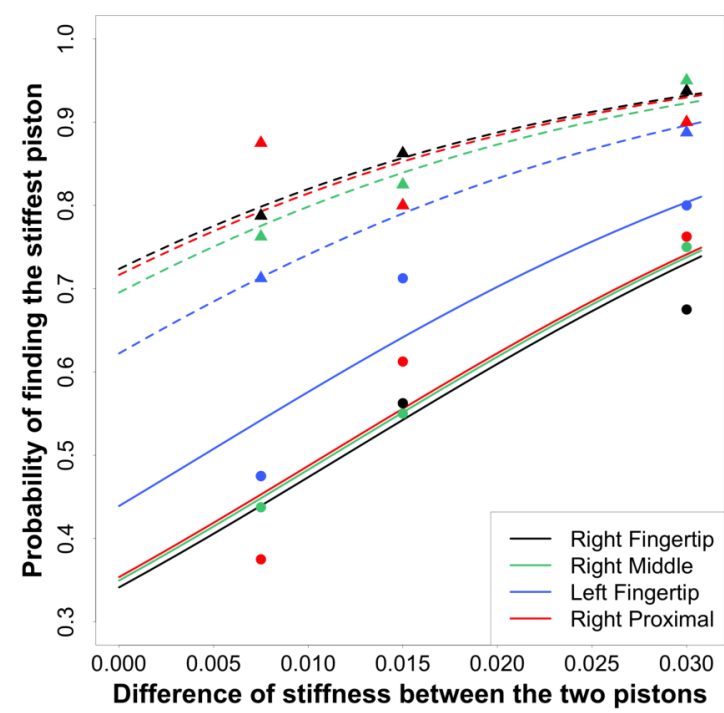

Figure 5: True discovery rate in function of the difference of stiffness between two pistons for the four different finger positions. Data are plot as points: triangle when our wearable device was considered as stiffer than the reference, circle otherwise. The plain/dashed lines represent the fitted curves to the data for each case.

We also analyzed the probability of finding the stiffest piston when there was no difference between the stiffness of the reference and the test pistons. We used a logistic regression model on the collected data to model the probability that the stiffest perceived piston was the one with the wearable device. We performed an analysis of deviance of the logistic regression model and we found a significant marginal effect for $\mathbf{C 3}(p<0.001)$. We performed a post-hoc analysis on the condition $\mathbf{C 3}$ using a Tukey test adapted to the logistic generalized regression model. We found a difference between the middle and the right finger tip positions $(Z=-4.46$, $p<0.001)$, as well as the left and right finger tip positions $(Z=$ $-2.90, p=0.019)$.

Psychometric curves. The four psychometric curves corresponding to the different stimulation locations (device positions) are shown on Figure 6. As can be observed, there is a clear offset: all these curves are shifted to the left of the point $(0,0.5)$. This means that the presence of the additional cutaneous stimulus has well increased the stiffness sensation. We could compute the Point of Subjective Equality (PSE) and the Just Noticeable Difference (JND) values for each curve. The results are: for the right fingertip (JND: $18.3 \%$, PSE: $-13.7 \%$ ), right intermediate phalanx (JND: $17.9 \%$, PSE: $-10.8 \%$ ), right proximal phalanx (JND: $17.7 \%$, PSE: $-11.9 \%$ ), and left fingertip (JND: $17.5 \%$, PSE: $-6.5 \%$ ). These JND values are similar to the magnitudes generally reported in previous psychophysical studies on stiffness perception [30], suggesting that the discrimination capability did not change drastically in our study. Then, the four PSEs are all negative, which still tends to validate our hypotheses $\mathrm{H} 1$ and $\mathrm{H} 2$ (and the increase in stiffness sensation due to the tactile cue). Moreover, the PSE and the perceived stiffness sensation seems to be lower in the case of the remote (left hand) finger condition compare to all the other (right hand) conditions. Our experiment had not been specifically designed to compute psychometric curves. The PSE and JND values provided here should be taken cautiously. A dedicated user study with advanced statistical analysis should be conducted to confirm these preliminary results.

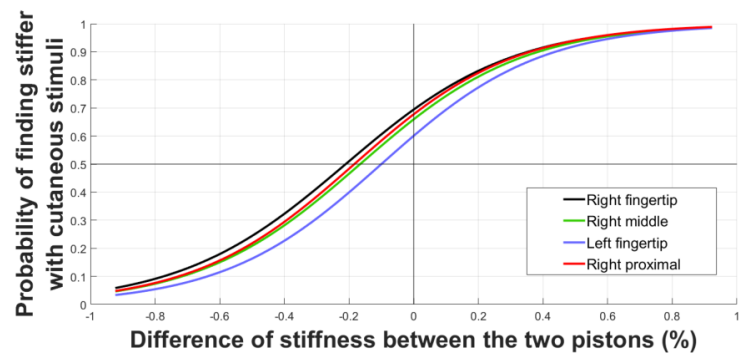

Figure 6: Psychometric curves for each location of the wearable device.

Subjective questionnaire. Figure 7 presents the answers collected through our subjective questionnaire (7-point Likert scale). Regarding the perception of the piston, participants reported that the piston barely seemed real $(\mathrm{Q} 1, M=4.75 ; S D=1.64)$. Regarding the contribution of the haptic device to the perception of stiffness, most of the participants agreed to this assumption $(\mathrm{Q} 2, M=5.63$; $S D=1.36$ ). The combination of both devices seem to contribute to this perception of stiffness (Q3, $M=5.56 ; S D=1.58)$. 5 participants gave the maximal rank. The participants did not particularly feel that the wearable device provided a higher contribution to the perception of stiffness $(\mathrm{Q} 4, M=4.38 ; S D=1.80)$.

Regarding the experiment, practicing was not also considered as considerably improving the association of both cutaneous and kinesthetic sensations (Q5, $M=5.06 ; S D=1.34$ ). Participants felt that the locations of the wearable device were highly influencing their perception of stiffness $(\mathrm{Q} 6, M=5.56 ; S D=1.59)$. Nine participants gave almost the minimal rank to the question 6 concerning the non-influence of the device locations. Finally, most of the participants reported overall medium levels of fatigue $(\mathrm{Q} 7, M=2.93$; $S D=1.56)$

Summary. The results of our perceptual evaluation show a significant effect of the stiffness perception when the wearable device was active. Both the objective measure and the subjective questionnaire confirm the increase of the stiffness perception when the haptic device and the wearable device are combined. Concerning the device positions on the fingers, there was no significant difference in the objective measure, but participants reported differences of stimuli in their answers to the questionnaire. The stimuli on the contralateral fingertip was however found less strong in the post-hoc analysis. 


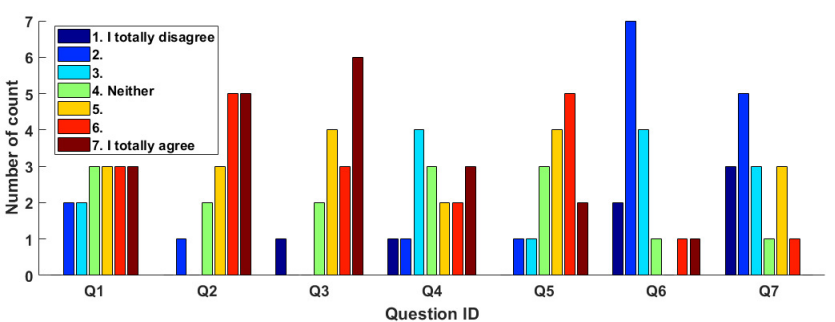

Figure 7: Bar-graph of the answers for each question. Q1: pressing real piston, $\mathrm{Q} 2$ : haptic device to perception, $\mathrm{Q} 3$ : combination to perception, Q4: tactile device's higher contribution, Q5: Practicing improves association, Q6: locations did not influence perception, Q7: Fatigue.

\section{USE CASES}

After the preliminary prototype of Sect. 3.2 and the quantitative evaluation of Sect. 4, we apply our approach to five use cases. Several passive and inexpensive tangible objects provide haptic information about the global shape/percept of the virtual objects, while our wearable haptic device provides haptic information about the dynamically changing mechanical property: the stiffness. A video showing these scenes can be found as supplemental material and at https://youtu.be/qA4xr81V4WA

\subsection{Setup}

Considering the results of Sect. 4, the wearable tactile device is worn on the user's index proximal phalanx. This configuration enables us to dynamically increase the perceived stiffness of the tangible objects, as detailed in Sect. 4.5, and, at the same time, it also enables the users to directly and naturally interact with the real environment using their fingertips.

The considered use cases in VR are shown in Table 1, and each tangible object is associated to a virtual one of similar shape. In these scenes, users wear either one or two wearable tactile devices on their proximal finger phalanges as well as a HTC Vive head-mounted display, which shows the virtual scene. Users sit or stand in front of a table on which tangible objects are laid down, and they are free to move their hand around. The motion of the user's hand is tracked using a Gametrak device, which registers the position of the volar side of the hand, and an inertial measurement unit (IMU), which registers its orientation (see also the bottom right picture of Table 1). These two pieces of information combined together enable us to reach a reasonably accurate tracking on the position and orientation of the user's hand. Similarly to [27], since we track the volar side of the hand, the user should not move the index finger with respect to the palm (i.e., IP and MCP joints should be fixed). A hand avatar mimicks the motion of the human hand in the virtual environment. The position of the hand avatar in the scene with respect to the virtual objects is carefully adjusted to match the position of the human hand with respect to the tangible objects. In this way, whenever the user touches one of the tangible objects in the real environment, the hand avatar touches the correspondent virtual object in the scene. The activation of the wearable device is then computed in real time, knowing the penetration depth in the virtual (and real) object as well as the given stiffness property. The virtual scene has been built using Unity 5.6, which renders the virtual scene and computes the contact interactions between the hand avatar and the virtual foams.

We also considered two AR use cases, shown in Table 2. The setup is the same as the one described above. However, this time, users are asked to wear a Microsoft HoloLens instead of the HTC Vive head-set, which projects a virtual scene onto the real environment. Users can therefore look directly at the real environment and at their own hand while the HoloLens superimposes the virtual objects onto the tangible ones.

\subsection{Use cases descriptions}

Use case \#1 in VR: inflating a balloon. The VR scene is composed of one rounded pump, a pressure indicator, and a balloon (see the first column of Table 1). The user is able to interact with the virtual pump which is superimposed to a deformable tangible object. Each time the pump is pressed, the displayed level of pressure rises, the balloon is inflated and, thus, the stiffness of the pump increases. These dynamic changes in the pump stiffness are provided through the wearable tactile device, by altering the perceived stiffness of the tangible pump, which does not change during the experience.

Use case \#2 in VR: launching two rockets. This use case is a follow-up of the previous one: the pressure system is the same, however the balloon is replaced by a set of rockets with launch buttons (see the second column of Table 1). The propulsion power of the rocket depends on the amount of pressure inserted into the system prior to launch. The second tangible object is a pushbutton. It acts as a spring with a hard and non deformable head whose perceived stiffness is also enhanced depending on the pressure. As before, these dynamic changes in pressure when interacting with the pumps and buttons are provided through the wearable tactile device.

Use case \#3 in VR: petting a rabbit. The VR scene is composed of a basket, a scale, and a rabbit (see the third column of Table 1). The users can move the rabbit from the basket to the scale and pet it by holding the tangible object, a simple cylinder made of foam, which coarsely resembles the rabbit. The rabbit breathing activity is simulated by inflating and deflating its abdomen, and it speeds up whenever the rabbit is lifted or moved too fast. As the rabbit breathes, its overall shape and stiffness vary. The user is able to feel these variations though two wearable tactile devices, worn on the right index proximal finger phalanx and on the right thumb proximal finger phalanx.

Use case \#4 in VR and AR: abdomen palpation. This use case, in VR, is shown in the fourth column of Table 1 . The scene is composed of a patient laying on a table inside a medical examination room. In front of the human user, we place a large parallelepiped made of foam, which coarsely resembles the abdomen of the patient and has constant stiffness. The user has to palpate the abdomen and locate a virtual cyst, simulated by a stiff sphere with a diameter of $3 \mathrm{~cm}$ [19]. When the fingertip avatar touches the simulated cyst-like sphere, the wearable tactile device is activated, rendering the increased stiffness of the area.

This palpation user case was also carried out in AR (see the first column of Table 2). The same VR scene described above is now superimposed to the real environment. The position of the superimposed virtual scene is carefully adjusted to match the position of the foam abdomen. Whenever the user touches the foam in the real environment, the user sees his hand touching the virtual abdomen and eventually feels an increase of stiffness.

Use case \#5 in AR: forearm palpation. The setup is shown in the second column of Table 2 . In this case, the tangible object is a real human forearm, placed on a table in front of the human user. The virtual scene is composed of two disks, superimposed to the human forearm, one near the wrist and one near the elbow. As before, the user wears one wearable tactile device on the right index proximal finger phalanx. Users are asked to palpate the real forearm and touch the two highlighted regions. As users palpate the forearm, their fingertip is directly in contact with the forearm skin. Whenever the fingertip touches one of the two highlighted regions, the wearable tactile device is activated, making the area feel stiffer.

\section{Discussion}

In our study, results showed a strong effect when wearing the active device, regardless of the locus of stimulation, i.e., the wearable haptic device did indeed increase the piston perceived stiffness. It is also interesting to note that users had the feeling that the position of the device holds importance. Some of them would prefer to have 


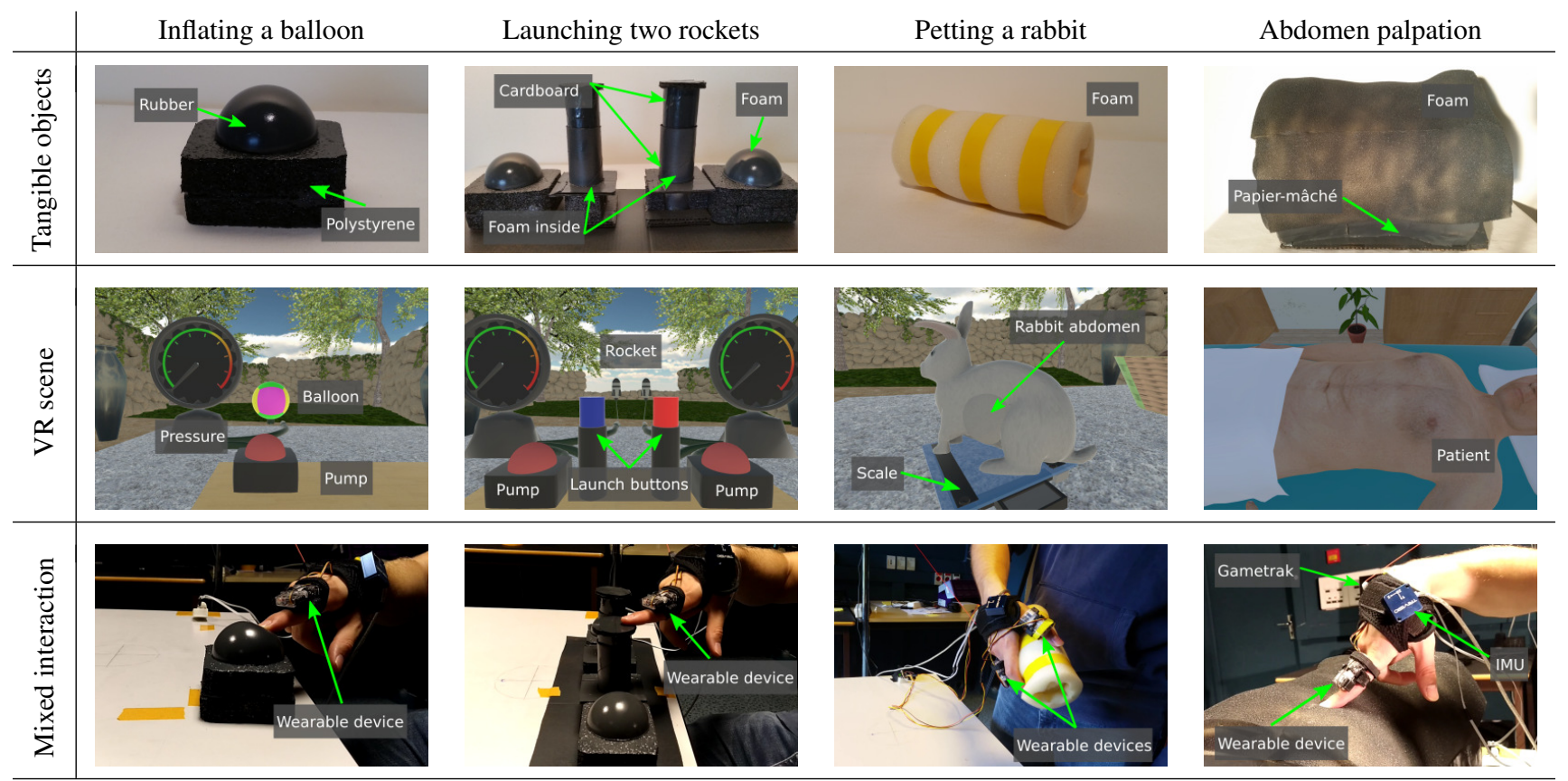

Table 1: Virtual Reality (VR) scenes. Each column refers to one VR use case. The first row shows the tangible objects employed, which are all passive and inexpensive. The second row shows the VR scene presented to the user through a HMD. The third row shows the user, wearing one or two wearable tactile devices, interacting with the tangible environment. The wearable devices are in charge of dynamically changing the perceived stiffness of the tangible objects according to what happens in the VR scene.

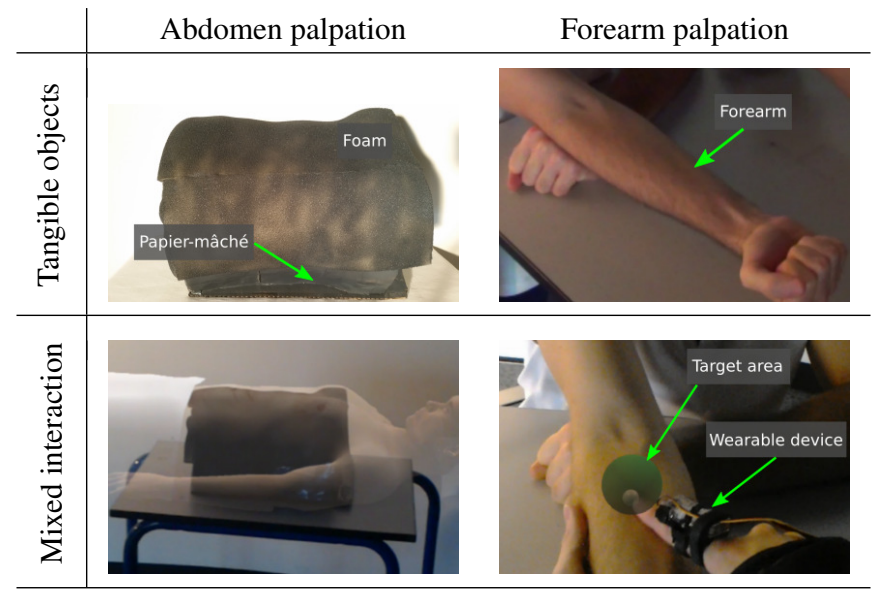

Table 2: Augmented Reality (AR) scenes. Each column refers to one AR use case. The first row shows the tangible objects employed. The second row shows the mixed-reality view, provided to the user through the Microsoft Hololens. The wearable device is in charge of dynamically changing the perceived stiffness of the tangible objects according to what happens in the AR scene.

it on the fingertip, while others would rather have it away from it. Despite this result, we found no significant difference between the considered loci of stimulation: fingertip, middle phalanx, proximal phalanx, or fingertip of the contralateral hand. However, the stiffness augmentation effect when providing tactile stimuli on the contralateral fingertip was found less strong than when providing stimuli on the finger interacting with the tangible object.

These results are quite promising. Indeed, retaining the stiffness alteration effect even when moving the tactile stimulus away from the contact point with the tangible object (i.e., the fingertip) opens up several interesting opportunities. For example, moving the wearable device toward the proximal finger phalanx leaves the fingertip free to directly interact with the tangible objects, providing a more natural haptic sensation. Touching tangible objects while wearing wearable devices on the fingertips has been in fact already proven to significantly reduce the effectiveness of AR systems [22]. Moreover, leaving the fingertip free can also improve the performance of model-based trackers [25].

In our work, we developed several use cases with stiff objects, always passive, whose stiffness was dynamically enhanced either globally or locally while pressing on them, holding them, or exploring them. Although the proposed approach is quite effective and promising, there are still several questions to be answered. For example, we can only increase the perceived stiffness of tangible objects, while it does not seem trivial to make them feel less stiff than they actually are. In this respect, an idea could be to start the interaction with the wearable device applying an initial force to the finger skin, and then partially release the belt when coming in contact with the tangible object. Since it is known that pressure mechanoreceptors tends to adapt to constant pressure stimuli [32], a timely decrease of pressure at the finger when touching the tangible object may decrease its perceived stiffness. Another interesting open question is how much we can increase the perceived stiffness of tangible objects via wearable haptics. Finally, as our wearable device is not able to effectively break contact with the finger skin, we were unable to take advantage of the effect of the temporal change of contact area, which is known to help stiffness perception $[9,29]$.

As for future work, our proof of concept could first be extended to other types of haptic sensation, such as for augmenting the perceived friction of tangible objects (as our 2-DoF wearable device can already provide skin stretch stimuli). Then, the use of a specific tracking system could enable to track the position of the user's hand and fingertips, e.g., Vicon Vero. Being able to directly track fingertips position should enable users to move their fingers freely with respect to the palm. Another promising idea is to modify the end-effector of our wearable device to make it wider and able to effectively making and breaking contact with the finger skin. As mentioned above, we expect this modification to significantly improve the delivery of stiffness stimuli, as stiffness perception is 
known to be influenced by the temporal change of contact area. Moreover, it could be interesting to combine the proposed approach with pseudo-haptics, in order to increase the range of stiffness levels that can be altered. Last, we also suggest carrying out further user studies to evaluate our approach in concrete applications such as with our use cases.

\section{CONCLUSION}

We introduced an innovative approach for VR and AR immersive environments, capable of dynamically altering the perceived stiffness of tangible objects by providing timely tactile stimuli through wearable haptic devices. This approach combines the haptic capabilities of both tangible objects and wearable haptics, delivering them through unobtrusive and inexpensive systems. Passive and uncomplicated tangible objects provide haptic information about the global shape/percept of the virtual objects, while wearable haptic devices provide haptic information about dynamically changing mechanical properties. We believe that these two pieces of haptic information combined together could significantly improve the effectiveness and immersiveness of haptic-enabled VR and AR experiences. We considered a representative proof-of-concept scenario, in which we used a 2-DoF wearable tactile device at the fingers. Whenever the user interacts with a tangible object during VR or AR experiences, the wearable device dynamically modifies its stiffness perception, making the tangible object feel more or less stiff depending on what is happening in the virtual scene. We used a wearable haptic device at the level of the proximal finger phalanx, and we could leave the user's fingertip free to directly interact with the tangible environment A user study and five use cases showed the potential and viability of our approach. Taken together our results pave the way for novel haptic systems in VR/AR applications better exploiting the multiple ways of providing simple and low-cost haptic displays.

\section{REFERENCES}

[1] S. Asano, S. Okamoto, and Y. Yamada. Vibrotactile stimulation to increase and decrease texture roughness. IEEE Trans. Human-Machine Systems, 45(3):393-398, 2015.

[2] Y. Ban, T. Kajinami, T. Narumi, T. Tanikawa, and M. Hirose. Modifying an identified curved surface shape using pseudo-haptic effect. In Proc. IEEE Haptics Symposium, pp. 211-216, 2012.

[3] M. Billinghurst, H. Kato, and I. Poupyrev. The magicbook-moving seamlessly between reality and virtuality. IEEE Computer Graphics and applications, 21(3):6-8, 2001.

[4] G. C. Burdea and P. Coiffet. Virtual reality technology, vol. 1. John Wiley \& Sons, 2003.

[5] A. D. Cheok, X. Yang, Z. Z. Ying, M. Billinghurst, and H. Kato. Touchspace: Mixed reality game space based on ubiquitous, tangible, and social computing. Personal and ubiquitous computing, 6(5-6):430-442, 2002.

[6] F. Chinello, C. Pacchierotti, M. Malvezzi, and D. Prattichizzo. A three revolute-revolute-spherical wearable fingertip cutaneous device for stiffness rendering. IEEE Transactions on Haptics, 2017.

[7] H. Culbertson and K. Kuchenbecker. Ungrounded haptic augmented reality system for displaying roughness and friction. IEEE/ASME Trans. Mechatronics, 22(4):1839-1849, 2017.

[8] S. Follmer, D. Leithinger, A. Olwal, A. Hogge, and H. Ishii. inform: dynamic physical affordances and constraints through shape and object actuation. In Proc. ACM UIST, vol. 13, pp. 417-426, 2013.

[9] K. Fujita and H. Ohmori. A new softness display interface by dynamic fingertip contact area control. In Proc. 5th World Multiconference on Systemics, Cybernetics and Informatics, pp. 78-82, 2001.

[10] A. Girard, M. Marchal, F. Gosselin, A. Chabrier, F. Louveau, and A. Lécuyer. Haptip: Displaying haptic shear forces at the fingertips for multi-finger interaction in virtual environments. Frontiers in ICT, 3:6, 2016.

[11] M. Harders, G. Bianchi, B. Knoerlein, and G. Székely. Calibration, registration, and synchronization for high precision augmented reality haptics. IEEE Trans. Visualization and Computer Graphics, 15(1):138149, 2009.
[12] D. Harley, A. P. Tarun, D. Germinario, and A. Mazalek. Tangible vr: Diegetic tangible objects for virtual reality narratives. In Proc. Conference on Designing Interactive Systems, pp. 1253-1263, 2017.

[13] V. Hayward, O. Astley, M. Cruz-Hernandez, D. Grant, and G. RoblesDe-La-Torre. Haptic interfaces and devices. Sensor Review, 24(1):1629, 2004.

[14] B. Insko. Passive haptics significantly enhances virtual environments. $\mathrm{PhD}$ thesis, University of North Carolina at Chapel Hill, 2001.

[15] H. Ishii, D. Leithinger, S. Follmer, A. Zoran, P. Schoessler, and J. Counts. Transform: Embodiment of radical atoms at milano design week. In Proc. ACM Conf. Human Factors in Computing Systems, pp. 687-694, 2015.

[16] I. Jang and D. Lee. On utilizing pseudo-haptics for cutaneous fingertip haptic device. In Proc. IEEE Haptics Symposium, pp. 635-639, 2014

[17] S. Jeon, S. Choi, and M. Harders. Rendering virtual tumors in real tissue mock-ups using haptic augmented reality. IEEE Trans. Haptics, 5(1):77-84, 2012

[18] S. Jeon, B. Knoerlein, M. Harders, and S. Choi. Haptic simulation of breast cancer palpation: A case study of haptic augmented reality. In Proc. IEEE ISMAR, pp. 237-238, 2010.

[19] S. Kodaganur, I. R. Hosamani, M. Doddamani, and K. Udaykumar. Mass in the left iliac fossaa diagnostic dilemma. Indian Journal of Surgery, 78(1):54-56, 2016.

[20] A. Lécuyer. Simulating haptic feedback using vision: A survey of research and applications of pseudo-haptic feedback. Presence: Teleoperators and Virtual Environments, 18(1):39-53, 2009.

[21] T. Maeda, R. Peiris, M. Nakatani, Y. Tanaka, and K. Minamizawa. Wearable haptic augmentation system using skin vibration sensor. In Proc. Virtual Reality International Conference, p. 25, 2016.

[22] M. Maisto, C. Pacchierotti, F. Chinello, G. Salvietti, A. De Luca, and D. Prattichizzo. Evaluation of wearable haptic systems for the fingers in augmented reality applications. IEEE Trans. Haptics, 2017.

[23] K. Minamizawa, S. Fukamachi, H. Kajimoto, N. Kawakami, and S. Tachi. Gravity grabber: wearable haptic display to present virtual mass sensation. In Proc. ACM SIGGRAPH emerging technologies, p. 8, 2007.

[24] T. Ohshima, K. Satoh, H. Yamamoto, and H. Tamura. Ar2 hockey system: A collaborative mixed reality system. Trans. VRSJ, 3(2):55-60, 1998.

[25] C. Pacchierotti, G. Salvietti, I. Hussain, L. Meli, and D. Prattichizzo. The hRing: a wearable haptic device to avoid occlusions in hand tracking. In Proc. IEEE Haptics Symposium, 2016.

[26] C. Pacchierotti, S. Sinclair, M. Solazzi, A. Frisoli, V. Hayward, and D. Prattichizzo. Wearable haptic systems for the fingertip and the hand: taxonomy, review, and perspectives. IEEE Trans. Haptics, 2017.

[27] C. Pacchierotti, A. Tirmizi, G. Bianchini, and D. Prattichizzo. Enhancing the performance of passive teleoperation systems via cutaneous feedback. IEEE Trans. Haptics, 8(4):397-409, 2015.

[28] C. Pacchierotti, A. Tirmizi, and D. Prattichizzo. Improving transparency in teleoperation by means of cutaneous tactile force feedback. ACM Trans. Applied Perception, 11(1):4, 2014.

[29] J. Park, J. Kim, Y. Oh, and H. Z. Tan. Compensation of perceived hardness of a virtual object with cutaneous feedback. In Proc. IEEE World Haptics Conference, pp. 101-106, 2017.

[30] W. M. B. Tiest and A. M. L. Kappers. Cues for haptic perception of compliance. IEEE Transactions on Haptics, 2(4):189-199, Oct 2009.

[31] Y. Zhao, L. H. Kim, Y. Wang, M. Le Goc, and S. Follmer. Robotic assembly of haptic proxy objects for tangible interaction and virtual reality, 102017.

[32] M. J. Zigler. Pressure adaptation-time: a function of intensity and extensity. The American Journal of Psychology, 44(4):709-720, 1932.

\section{ACKNOWLEDGMENTS}

This research has received funding from Rennes Métropole under the "Allocation d'Installation Scientifique (AIS) 2017" programme. 